\title{
Large time behavior of solutions to degenerate parabolic equations
}

\author{
Shaohua Chen and Runzhang Xu
}

\begin{abstract}
This paper deals with large time behavior of the Dirichlet problem to the degenerate parabolic equation $u_{t}=g(u) \Delta u+f(u)$ in a bounded domain $\Omega \subset R^{n}$ with smooth boundary $\partial \Omega$. Under suitable conditions on $f(u)$ and $g(u)$, we show that all solutions will converge to the steady state exponentially.
\end{abstract}

Mathematics Subject Classification. 35K55, 35K65, 35B40.

Keywords. Degenerate parabolic equations, Steady state, Exponential convergence.

We study the long time behavior of global solutions to the following degenerate parabolic equation:

$$
\left\{\begin{array}{l}
u_{t}=g(u) \Delta u+f(u), \\
u(x, 0)=u_{0}(x), \\
\left.u\right|_{\partial \Omega}=0,
\end{array}\right.
$$

where $\Omega \subset R^{n}$ is a bounded domain with smooth boundary $\partial \Omega$. The global existence of the classical solutions has been proved by many authors under various conditions on $f$ and $g$, see $[1-4,8,9,11]$.

Winkler [9] considered the following problem:

$$
\left\{\begin{array}{l}
u_{t}=f(u)(\Delta u+g(u)), \\
u(x, 0)=u_{0}(x), \\
\left.u\right|_{\partial \Omega}=0
\end{array}\right.
$$

in a bounded domain $\Omega$ with smooth boundary, where $f(0)=0$. He showed that all solutions approach to the steady state as $t \rightarrow \infty$ if $g(u)$ satisfies

$$
\lim \inf _{s \rightarrow 0} \frac{g(s)}{s}>\lambda_{1} \quad \text { and } \quad \lim \sup _{s \rightarrow \infty} \frac{g(s)}{s}<\lambda_{1}
$$

where $\lambda_{1}$ is the first eigenvalue of $-\Delta$ with zero Dirichlet boundary condition. He also discussed the stability of the steady states and the properties of the 
$\omega$-set. He [10] also discussed large time behavior of positive solution for the equation $u_{t}=u^{p} \Delta u-u^{q}$ with $p, q \geq 1$.

Zhou et al. dealt with the following degenerate parabolic equation with source and absorption:

$$
\left\{\begin{array}{l}
u_{t}=u^{p} \Delta u+a u^{q}-b u^{r}, \\
u(x, 0)=u_{0}(x), \\
\left.u\right|_{\partial \Omega}=0,
\end{array}\right.
$$

in a bounded domain $\Omega$ with smooth boundary, where $p \geq 1, q>0, r>1, a, b \geq$ 0 . They proved that the global solutions uniformly tend to the positive steady state of the problem as $t \rightarrow \infty$ if either $q<p+1 \leq r$ or $p-1<q<r<p+1$. They also established the uniform asymptotic profiles for the decay solutions when the problem is governed by the nonlinear diffusion of absorption.

We first consider the problem (1) and list the sufficient conditions such that all solutions converge to the corresponding steady state as $t \rightarrow \infty$ exponentially. Then we give two examples to verify the sufficient conditions. To our knowledge, such convergence is reported very limited in literature, see [6].

Throughout this paper, we denote by $\lambda_{1}$ and $\psi$ the first eigenvalue and eigenfunction of

$$
\Delta \psi+\lambda_{1} \psi=0 \quad \text { with }\left.\quad \psi\right|_{\partial \Omega}=0 .
$$

Theorem 1. Assume that

1. Problem (1) has a classical solution $u(x, t)$ with $u(x, t) / \psi(x)$ bounded in $(0, \infty)$ and $\phi(x)$ is the corresponding positive steady state, that is,

$$
\left\{\begin{array}{l}
g(\phi) \Delta \phi+f(\phi)=0 \\
\left.\phi\right|_{\partial \Omega}=0
\end{array}\right.
$$

with $\phi \in C^{1}(\bar{\Omega}) \bigcap C^{2}(\Omega)$ and $\phi(x) \geq c_{0} \operatorname{dist}(x, \partial \Omega)$ for all $x \in \Omega$ and $c_{0}>0$.

2. $g(u)>0$ for $u>0, g(0)=0, g^{\prime \prime}(u)$ exists, and

$$
\frac{u(x, t) g[u(x, t)] f[\phi(x)]-\phi(x) g[\phi(x)] f[u(x, t)]}{[u(x, t)-\phi(x)] g[\phi(x)]} \geq \varepsilon \phi(x),
$$

for some small $\varepsilon>0$ and any $(x, t) \in \Omega \times[0, \infty)$.

3. $u_{0} \in C^{2+\mu}(\bar{\Omega})$ for some $\mu \in(0,1), u_{0}(x)>0$ in $\Omega$ and $\left.u_{0}(x)\right|_{\partial \Omega}=0$.

Then, there exists a constant $C>0$ such that

$$
|u(x, t)-\phi(x)| \leq C \phi(x) e^{-\varepsilon t / 2} .
$$

Proof. For any even number $n$, define

$$
w_{n}(t)=\int_{\Omega} \phi(x) y^{n}(t, x) d x, \quad \text { and } \quad y(t, x)=\frac{u(x, t)}{\phi(x)}-1 .
$$

Notice that, $u(x, t) / \phi(x)$ is bounded because both $u(x, t) / \psi(x)$ and $\psi(x) / \phi(x)$ are bounded in $\Omega$. If $Q \in \partial \Omega$, then,

$$
\lim _{x \rightarrow Q} \frac{u(x, t)}{\phi(x)}=\lim _{x \rightarrow Q} \frac{u(x, t)-u(Q, t)}{\phi(x)-\phi(Q)}=\frac{\partial u(Q, t) / \partial \mathbf{n}}{\partial \phi(Q) / \partial \mathbf{n}},
$$


where $\mathbf{n}$ is the outer normal direction. By Assumption $1, \partial \phi / \partial \mathbf{n}>0$ on $\partial \Omega$. Thus $u(x, t) / \phi(x)$ is continuous on $\bar{\Omega}$ after boundary extension and (5) is well defined.

Taking the derivative of $w_{n}(t)$ in $(5)$ and integrating by parts, we obtain

$$
\begin{aligned}
w_{n}^{\prime}(t)= & n \int_{\Omega} y^{n-1}[g(u) \Delta u+f(u)] d x \\
= & -n(n-1) \int_{\Omega} y^{n-2} g(u) \nabla y \nabla u d x \\
& -n \int_{\Omega} y^{n-1} g^{\prime}(u)|\nabla u|^{2} d x+n \int_{\Omega} y^{n-1} f(u) d x .
\end{aligned}
$$

Notice that the boundary integration is 0 because $\left.g(u)\right|_{\partial \Omega}=0$. Since

$$
\nabla u=\phi \nabla\left(\frac{u}{\phi}\right)+\frac{u}{\phi} \nabla \phi=\phi \nabla y+\frac{u}{\phi} \nabla \phi,
$$

the first term of (6) can be written as

$$
\begin{aligned}
\text { The first term }= & -n(n-1) \int_{\Omega} \phi y^{n-2} g(u)|\nabla y|^{2} d x \\
& -n(n-1) \int_{\Omega} \phi^{-1} y^{n-2} u g(u) \nabla y \nabla \phi d x .
\end{aligned}
$$

Integrating by parts for the second term of (8) yields

$$
\begin{aligned}
& -n \int_{\Omega} \phi^{-1} u g(u) \nabla\left[y^{n-1}\right] \nabla \phi d x=-n \int_{\Omega} \phi^{-2} y^{n-1} u g(u)|\nabla \phi|^{2} d x \\
& +n \int_{\Omega} \phi^{-1} y^{n-1} g(u) \nabla u \nabla \phi d x+n \int_{\Omega} \phi^{-1} y^{n-1} u g^{\prime}(u) \nabla u \nabla \phi d x \\
& \quad+n \int_{\Omega} \phi^{-1} y^{n-1} u g(u) \Delta \phi d x .
\end{aligned}
$$

Substituting (8) and (9) into (6), we have

$$
\begin{aligned}
w_{n}^{\prime}(t)= & -n(n-1) \int_{\Omega} \phi y^{n-2} g(u)|\nabla y|^{2} d x-n \int_{\Omega} \phi^{-2} y^{n-1} u g(u)|\nabla \phi|^{2} d x \\
& +n \int_{\Omega} \phi^{-1} y^{n-1} g(u) \nabla u \nabla \phi d x+n \int_{\Omega} \phi^{-1} y^{n-1} u g^{\prime}(u) \nabla u \nabla \phi d x \\
& +n \int_{\Omega} \phi^{-1} y^{n-1} u g(u) \Delta \phi d x-n \int_{\Omega} y^{n-1} g^{\prime}(u)|\nabla u|^{2} d x \\
& +n \int_{\Omega} y^{n-1} f(u) d x .
\end{aligned}
$$

Using (7) again in the third and the sixth term of (10), we obtain

$$
\begin{aligned}
n \int_{\Omega} \phi^{-1} y^{n-1} g(u) \nabla u \nabla \phi d x= & n \int_{\Omega} y^{n-1} g(u) \nabla y \nabla \phi d x \\
& +n \int_{\Omega} \phi^{-2} u y^{n-1} g(u)|\nabla \phi|^{2} d x
\end{aligned}
$$


and

$$
\begin{aligned}
-n \int_{\Omega} y^{n-1} g^{\prime}(u)|\nabla u|^{2} d x= & -n \int_{\Omega} \phi y^{n-1} g^{\prime}(u) \nabla u \nabla y d x \\
& -n \int_{\Omega} \phi^{-1} y^{n-1} u g^{\prime}(u) \nabla u \nabla \phi d x .
\end{aligned}
$$

Hence, we have

$$
\begin{aligned}
w_{n}^{\prime}(t)= & -n(n-1) \int_{\Omega} \phi y^{n-2} g(u)|\nabla y|^{2} d x+n \int_{\Omega} y^{n-1} g(u) \nabla y \nabla \phi d x \\
& +n \int_{\Omega} \phi^{-1} y^{n-1} u g(u) \Delta \phi d x-n \int_{\Omega} \phi y^{n-1} g^{\prime}(u) \nabla u \nabla y d x \\
& +n \int_{\Omega} y^{n-1} f(u) d x,
\end{aligned}
$$

after cancelling four terms. Using (7) again in the fourth term of (13) and integrating by parts, we have

the second term + the fourth term in $(13)=n \int_{\Omega} y^{n-1} g(u) \nabla y \nabla \phi d x$

$$
\begin{aligned}
& -n \int_{\Omega} \phi^{2} y^{n-1}|\nabla y|^{2} g^{\prime}(u) d x-n \int_{\Omega} y^{n-1} u g^{\prime}(u) \nabla y \nabla \phi d x \\
= & -n \int_{\Omega} \phi y^{n-2}(u-\phi)|\nabla y|^{2} g^{\prime}(u) d x \\
& +n \int_{\Omega} y^{n-1}\left[g(u)-u g^{\prime}(u)\right] \nabla y \nabla \phi d x \\
= & -n \int_{\Omega} \phi y^{n-2}(u-\phi)|\nabla y|^{2} g^{\prime}(u) d x \\
& -\int_{\Omega} y^{n}\left[g(u)-u g^{\prime}(u)\right] \Delta \phi d x+\int_{\Omega} y^{n} u g^{\prime \prime}(u) \nabla u \nabla \phi d x .
\end{aligned}
$$

For any $\varepsilon>0$, choose the even number $n$ sufficiently large such that

$$
\left|(u-\phi) g^{\prime}(u)\right| \leq(n-1) g(u), \quad\left|\left[g(u)-u g^{\prime}(u)\right] \Delta \phi / \phi\right| \leq \frac{\varepsilon n}{4},
$$

and

$$
2 \beta u|\nabla \phi| / \phi \leq \frac{\varepsilon n}{4} .
$$

Substituting (2), (14) and (15) into (13) yields

$$
\begin{aligned}
w_{n}^{\prime}(t) & \leq \frac{\varepsilon n}{2} w_{n}(t)-n \int_{\Omega} y^{n-1} \phi^{-1} \frac{u g(u) f(\phi)-\phi g(\phi) f(u)}{g(\phi)} d x \\
& =\frac{\varepsilon n}{2} w_{n}(t)-n \int_{\Omega} y^{n} \frac{u g(u) f(\phi)-\phi g(\phi) f(u)}{(u-\phi) g(\phi)} d x \\
& \leq-\frac{\varepsilon n}{2} w_{n}(t),
\end{aligned}
$$

where we used the assumption (3). Solving the inequality (16), we have

$$
w_{n}(t) \leq w_{n}(0) e^{-\varepsilon n t / 2} .
$$


Taking $n^{\text {th }}$ roots and letting $n \rightarrow \infty$, we obtain

$$
\max _{\bar{\Omega}}\left|\frac{u(x, t)}{\phi(x)}-1\right| \leq \max _{\bar{\Omega}}\left|\frac{u_{0}(x)}{\phi(x)}-1\right| e^{-\varepsilon t / 2} .
$$

Hence, (4) holds. This completes the proof.

Remark 1. It is easy to see that the positive steady state of (1) is unique because (4) is true for all initial values $u_{0}(x)$ which satisfies Assumption 3 in Theorem 1.

Example 1. Consider the following equation

$$
\left\{\begin{array}{l}
u_{t}=\left(\alpha u+\beta u^{2}\right) \Delta u+u\left(a+u^{r}\right) \\
u(x, 0)=u_{0}(x), \\
\left.u\right|_{\partial \Omega}=0
\end{array}\right.
$$

where $\alpha, \beta, a \geq 0$ and $0<r \leq 2, u_{0}(x) \geq c_{0} \operatorname{dist}(x, \partial \Omega)$ for all $x \in \Omega$ and $c_{0}>0$. If one of the following conditions is satisfied

I. $\quad a \alpha>0$ and $r \leq 1$,

II. $a \alpha>0,1<r<2$ and $\frac{\alpha^{r}}{4 \beta^{r}}\left(\frac{r-1}{2(2-r)}\right)^{r-1}<a$,

then (3) holds. In fact, by [1], the positive classical solution exists and $u(x, t) /$ $\psi(x) \leq M$ for some $M>0$ and all $t>0$. It is easy to see that the corresponding steady state exists. Now we verify (3), which can be written as

$$
\begin{aligned}
\frac{u g(u) f(\phi)-\phi g(\phi) f(u)}{(u-\phi) g(\phi)}= & \frac{u\left[\left(\alpha u+\beta u^{2}\right)\left(a+\phi^{r}\right)-\left(\alpha \phi+\beta \phi^{2}\right)\left(a+u^{r}\right)\right]}{(u-\phi)(\alpha+\beta u)} \\
= & \frac{u}{\alpha+\beta u}\left[a \alpha+a \beta(u+\phi)+\alpha \frac{u \phi^{r}-\phi u^{r}}{u-\phi}\right. \\
& \left.+\beta u^{r} \phi^{r} \frac{u^{2-r}-\phi^{2-r}}{u-\phi}\right] .
\end{aligned}
$$

Under condition I, we can simplify (18) as follows:

$$
\begin{array}{r}
\frac{u}{\alpha+\beta u}\left[a \alpha+a \beta(u+\phi)+\alpha(1-r) u^{r} \phi^{r} \int_{0}^{1} v^{-r}(\theta) d \theta\right. \\
\left.+\beta(2-r) u^{r} \phi^{r} \int_{0}^{1} v^{1-r}(\theta) d \theta\right] \geq \frac{a \alpha u}{\phi(\alpha+\beta u)} \phi,
\end{array}
$$

where $v(\theta)=\theta u+(1-\theta) \phi$. It is easy to see that $\varepsilon \psi(x)$ is a sub-solution to (17) for sufficiently small $\varepsilon>0$. So $u / \phi=(u / \psi)(\psi / \phi) \geq \varepsilon \min (\psi / \phi) \triangleq \varepsilon_{0}>0$, we have shown that (3) holds.

Under condition II, we can simplify (18) as follows:

$$
\begin{aligned}
\frac{u}{\alpha+\beta u}[a \alpha+ & 2 a \beta \int_{0}^{1} v(\theta) d \theta-\frac{\alpha u \phi\left(u^{r-1}-\phi^{r-1}\right)}{u-\phi} \\
& \left.+\beta(2-r)(u \phi)^{r} \int_{0}^{1} \frac{1}{v^{r-1}(\theta)} d \theta\right] .
\end{aligned}
$$


Using the inequality

$$
\frac{u^{p+q}-\phi^{p+q}}{u^{p}-\phi^{p}} \geq \frac{p+q}{p}(u \phi)^{q / 2},
$$

for any $p, q \geq 0$ (see [7]), we obtain

$$
\frac{u-\phi}{u^{r-1}-\phi^{r-1}} \geq \frac{1}{r-1}(u \phi)^{(2-r) / 2},
$$

which can be written as

$$
\frac{u^{r-1}-\phi^{r-1}}{u-\phi} \leq(r-1)(u \phi)^{(r-2) / 2} .
$$

By Cauchy inequality,

$$
\begin{aligned}
& \left|\frac{\alpha u \phi\left(u^{r-1}-\phi^{r-1}\right)}{u-\phi}\right| \leq \alpha(r-1)(u \phi)^{r / 2} \\
& \quad=\alpha \int_{0}^{1}(u \phi)^{r / 2} v^{-(r-1) / 2}(\theta) \cdot v^{(r-1) / 2}(\theta) d \theta \\
& \quad \leq \int_{0}^{1}\left\{\beta(2-r)(u \phi)^{r} v^{1-r}(\theta)+\frac{\alpha^{2}}{4 \beta(2-r)} v^{r-1}(\theta)\right\} d \theta .
\end{aligned}
$$

Using Young inequality

$$
a \cdot b \leq \frac{c}{p} a^{p}+\frac{1}{q c^{q / p}} b^{q}, \quad \frac{1}{p}+\frac{1}{q}=1,
$$

with $p=1 /(r-1), q=1 /(2-r)$ and $c=2 a \beta /(r-1)$, we obtain,

$$
\begin{aligned}
& \frac{\alpha^{2}}{4 \beta(2-r)} \int_{0}^{1} v^{r-1}(\theta) d \theta \leq 2 a \beta \int_{0}^{1} v(\theta) d \theta \\
& \quad+\frac{2-r}{[2 a \beta /(r-1)]^{(r-1) /(2-r)}}\left(\frac{\alpha^{2}}{4 \beta(2-r)}\right)^{1 /(2-r)} \\
& =2 a \beta \int_{0}^{1} v(\theta) d \theta+\left(\frac{(2-r)^{2-r}}{[2 a \beta /(r-1)]^{r-1}} \frac{\alpha^{2}}{4 \beta(2-r)}\right)^{1 /(2-r)} \\
& =2 a \beta \int_{0}^{1} v(\theta) d \theta+\left[\frac{\alpha^{2}}{4 \beta^{r}}\left(\frac{r-1}{2 a(2-r)}\right)^{r-1}\right]^{1 /(2-r)} .
\end{aligned}
$$

By Condition II, we can find an $\varepsilon_{1}>0$ such that

$$
\frac{\alpha^{2}}{4 \beta^{r}}\left(\frac{r-1}{2(2-r)}\right)^{r-1}=a\left(\alpha-\alpha \varepsilon_{1}\right)^{2-r},
$$

which implies that

$$
\begin{aligned}
{\left[\frac{\alpha^{2}}{4 \beta^{r}}\left(\frac{r-1}{2 a(2-r)}\right)^{r-1}\right]^{1 /(2-r)} } & =\left[a\left(\alpha-\alpha \varepsilon_{1}\right)^{2-r}\left(\frac{1}{a}\right)^{r-1}\right]^{1 /(2-r)} \\
& =a\left(\alpha-\alpha \varepsilon_{1}\right)
\end{aligned}
$$


or

$$
\begin{aligned}
-\frac{\alpha u \phi\left(u^{r-1}-\phi^{r-1}\right)}{u-\phi} \geq & -a \alpha+a \alpha \varepsilon_{1}-2 a \beta \int_{0}^{1} v(\theta) d \theta \\
& -\beta(2-r)(u \phi)^{r} \int_{0}^{1} \frac{1}{v^{r-1}(\theta)} d \theta .
\end{aligned}
$$

Substituting (22) and (23) into (19) we have

$$
\frac{u g(u) f(\phi)-\phi g(\phi) f(u)}{(u-\phi) g(\phi)} \geq \frac{u}{\alpha+\beta u} a \alpha \varepsilon_{1} .
$$

Hence (3) holds.

Example 2. Consider the following equation

$$
\left\{\begin{array}{l}
u_{t}=u^{p} \Delta u+a u^{q}+b u^{r} \\
u(x, 0)=u_{0}(x), \\
\left.u\right|_{\partial \Omega}=0
\end{array}\right.
$$

where $a>0, q \leq 1,0<p<q+1, u_{0}(x) \geq \operatorname{cdist}(x, \partial \Omega)$ for all $x \in \Omega$ and $c>0$. If one of the following conditions is satisfied

I. $q<r<p+1$ and $b>0$,

II. $r=p+1, b>0$ and $\lambda_{1}>1$,

III. $r \geq p+1$ and $b<0$,

then (3) holds. In fact, by [1] and [11], the positive classical solution exists for all $t>0, \varepsilon_{0} \leq u(x, t) / \psi(x) \leq M$, and by [5], the corresponding steady state exists. Under Condition I and II, (3) can be written as

$$
\begin{aligned}
\frac{u g(u) f(\phi)-\phi g(\phi) f(u)}{(u-\phi) g(\phi)}= & \frac{u^{q}}{\phi^{p-q}}\left[a \frac{u^{p+1-q}-\phi^{p+1-q}}{u-\phi}\right. \\
& \left.+b(p+1-r) \phi^{r-q} u^{r-q} \int_{0}^{1} v^{p-r}(\theta) d \theta\right] \\
\geq & a \frac{u^{q}}{\phi^{p-q}} \frac{u^{p+1-q}-\phi^{p+1-q}}{u-\phi}
\end{aligned}
$$

If $p \geq q$, using (20), we have

$$
\begin{aligned}
a \frac{u^{q}}{\phi^{p-q}} \frac{u^{p+1-q}-\phi^{p+1-q}}{u-\phi} & \geq a(p+1-q)\left(\frac{u}{\phi}\right)^{(p+q) / 2} \phi^{q} \\
& \geq a(p+1-q)\left(\frac{u}{\phi}\right)^{(p+q) / 2} \frac{\phi}{\max \left(\phi^{1-q}\right)} \geq \varepsilon \phi,
\end{aligned}
$$

for some small $\varepsilon>0$. If $p<q$,

$$
\begin{gathered}
a \frac{u^{q}}{\phi^{p-q}} \frac{u^{p+1-q}-\phi^{p+1-q}}{u-\phi}=a(p+1-q) \frac{u^{q}}{\phi^{p-q}} \int_{0}^{1} \frac{1}{[\theta u+(1-\theta) \phi]^{q-p}} d \theta \\
\geq a(p+1-q)\left(\frac{u}{\phi}\right)^{p} \int_{0}^{1} \frac{1}{\max [\theta+(1-\theta) \phi / u]^{q-p}} d \theta \frac{1}{\max \phi^{1-q}} \phi .
\end{gathered}
$$

Since $\phi / u$ is bounded, we have verified that (3) holds. 
Under Condition III, (3) can be written as

$$
\begin{aligned}
\frac{u g(u) f(\phi)-\phi g(\phi) f(u)}{(u-\phi) g(\phi)}= & \frac{u^{q}}{\phi^{p-q}}\left[a(p+1-q) \int_{0}^{1} v^{p-q}(\theta) d \theta\right. \\
& \left.-b(r-p-1) \phi^{p+1} u^{p+1} \int_{0}^{1} v^{r-p-2}(\theta) d \theta\right] \\
\geq & a(p+1-q)\left(\frac{u}{\phi}\right)^{(p+q) / 2} \frac{\phi}{\max \left(\phi^{1-q}\right)} \geq \varepsilon \phi .
\end{aligned}
$$

\section{Acknowledgements}

This work was supported by the NSERC (Canada, RGPIN 251200-2008), the National Natural Science Foundation of China (11101102), China Postdoctoral Science Foundation(2013M540270), Heilongjiang Postdoctoral Foundation (LBH-Z13056), the Support Plan for the Young College Academic Backbone of Heilongjiang Province (1252G020) and Fundamental Research Funds for the Central Universities. The authors would like to thank the referees for their valuable suggestions which greatly improve the presentation of this article.

\section{References}

[1] Chen, S.: Boundedness and blowup for nonlinear degenerate parabolic equations. Nonlinear Anal. 70(2), 1087-1095 (2009)

[2] Chen, S., Yu, D.: Global existence and blowup solutions for quasilinear parabolic equations. J. Math. Anal. Appl. 335, 151-167 (2007)

[3] Friedman, A., McLeod, B.: Blow-up of solutions of nonlinear degenerate parabolic equations. Arch. Rational Mech. Anal. 96(1), 55-80 (1986)

[4] Ghergu, M.: Large time behavior of solutions to degenerate parabolic equations with weights. J. Math. Anal. Appl. 352(1), 132-138 (2009)

[5] Hernndez, J., Mancebo, F., Vega, J.: Positive solutions for singular nonlinear elliptic equations. Proc. Roy. Soc. Edinburgh Sect. A 137(1), 41-62 (2007)

[6] Madeira, G.F., do Nascimento, A.S.: Exponentially stable equilibria to an indefinite nonlinear Neumann problem in smooth domains. NoDEA Nonlinear Differ. Equ. Appl. 18(5), 599-614 (2011)

[7] Qi, F., Xu, S.: Refinements and extensions of an inequality II. J. Math. Anal. Appl. 211(2), 616-620 (1997)

[8] Wiegner, M.: A degenarate difussion equation with a nonlinear source term. Nonlinear Anal. TMA 28, 1977-1995 (1997)

[9] Winkler, M.: Large time behavior and stability of equilibria of degenerate parabolic equations. J. Dyn. Differ. Equ. 17(2), 331-351 (2005) 
[10] Winkler, M.: Large time behavior of solutions to degenerate parabolic equations with absorption. NoDEA Nonlinear Differ. Equ. Appl. 8(3), 343-361 (2001)

[11] Zhou, S., Bai, X., Zheng, S.: Large time behavior of solutions to a degenerate parabolic equation not in divergence form. J. Math. Anal. Appl. 373(1), 252263 (2011)

Shaohua Chen

Department of Mathematics

Physics and Geology

Cape Breton University

Sydney

NS B1P 6L2

Canada

e-mail: george_chen@cbu.ca

Runzhang Xu

College of Science

Harbin Engineering University

Harbin 150001

People's Republic of China

e-mail: xurunzh@163.com

Received: 28 February 2014.

Accepted: 13 July 2014. 